\title{
Isolated angiitis of central nervous system with pleocytosis in the cerebrospinal fluid
}

\author{
Henrique B Ferraz, José Augusto B Rolim, Afonso C Neves, Luiz Augusto F Andrade, \\ José F Rodrigues, Joao Norberto Stavale
}

\begin{abstract}
Summary
We report the case of a 32-year-old woman with isolated angiitis of the central nervous system. This case shows that high levels of pleocytosis may not rule out isolated angiitis of the central nervous system if this diagnosis can be considered on clinical grounds.
\end{abstract}

Keywords: angiitis; central nervous system; vasculitis cerebrospinal fluid; meningitis

Isolated angiitis of the central nervous system (IACNS) is a rare neurological disorder characterised by acute progressive headache, focal neurological deficits and a poor prognosis. The cerebrospinal fluid (CSF) may be normal or show mild to moderate inflammatory changes with pleocytosis and increased protein levels. Diagnosis is based on a clinical picture of neurological deficits associated with headache and the sparing of systemic vasculature. The angiograms may show suggestive abnormalities but only cerebral histopathologic examination can confirm the diagnosis. We present the case of a patient with isolated angiitis of the central nervous system, confirmed by autopsy, in whom a mistaken diagnosis of central nervous system (CNS) infection was made due to high pleocytosis in the CSF.

\section{Case report}

A 32-year-old black woman was admitted with a three-day history of severe throbbing headache. The headache worsened with loud sounds and light. She experienced a progressive increase of pain and, on the day of the admission, developed sudden paralysis of the left side of the body and difficulty in speech and swallowing. She had been taking birth control pills for seven months. She had had meningitis five years before. There was no fever or other general symptoms. Her clinical examination was completely normal, including blood pressure and cardiac auscultation. The neurological examination revealed normal mentation with paralysis of left leg, multiple nystagmus, left cerebellar ataxia, right peripheral facial paresis, and paresis of IX, X, XI and XII cranial nerves on the left. The deep tendon reflexes were normal and plantar responses were both absent.
Computed tomography (CT) of the brain was normal. A CSF examination revealed 637 leucocytes $/ \mathrm{mm}^{3}$ with a differential of $80 \% \mathrm{lym}$ phocytes, $8 \%$ monocytes and $12 \%$ neutrophils, protein $10.5 \mathrm{~g} / 1$ and glucose $8.1 \mathrm{~g} / 1$. Bacterioscopy and fungal examination, serologic tests for syphilis and cysticercosis, and CSF culture were all negative. Blood count, rheumatological tests, including serum complement, and biochemical work-up were all normal.

Her level of consciousness progressively deteriorated and clinical and radiological signs of pulmonary infection developed. Ceftriaxone $2 \mathrm{~g} /$ day and dexamethasone $24 \mathrm{mg} /$ day were administered in the second day. The patient died on the 13th day of admission due to Staphylococcus aureus infection. Autopsy revealed pulmonary infection, septicaemia and histopathologic changes of 'granulomatous angiitis' confined to the pons and medulla oblongata. The leptomeninges had infiltration of lymphocytes and monocytes and no giant cells were seen. The histological changes in the brainstem consisted of fibrinoid necrosis of the wall of arterioles and venules, with infiltration of polymorphonuclear leucocytes, lymphocytes and monocytes (figure). No multinucleated giant cells or granuloma formation were found. The examination of tissues outside the CNS did not reveal evidence of focal vasculitis or granulomatous lesions.

\section{Discussion}

The early institution of steroids and immunosuppressants, such as azathioprine and cyclophosphamide, can substantially improve the prognosis of IACNS. ${ }^{12}$ The diagnosis should therefore be considered in every young patient

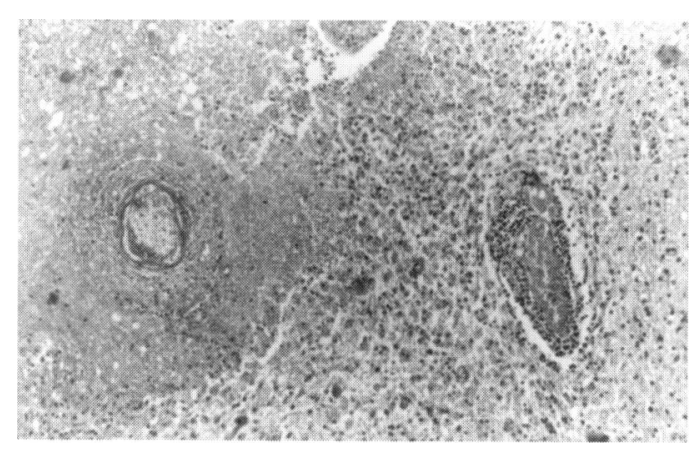

Figure Angiitis of the pons with lymphocytic infiltrate and fibrinoid necrosis 
with acute headache and focal neurological signs. The diagnosis is, however, difficult to make without leptomeningeal and cerebral biopsy. Neuroimaging studies may often be normal and the diagnosis must often be based on clinical and laboratory findings, since cerebral and leptomeningeal biopsy is not a routine or desirable diagnostic procedure.

CSF abnormalities in IACNS are due to the inflammatory process. Calabrese and Mallek, in one of the largest series of IACNS patients in the literature (48 patients), observed pleocytosis in 27 of $40(68 \%)$ cases in whom CSF was examined. ${ }^{1}$ In this study, the highest pleocytosis observed was 330 cells $/ \mathrm{mm}^{3}$, and the average was 60 cells $/ \mathrm{mm}^{3}$. Mononuclear cells predominated and the protein had an average level of $11.8 \mathrm{~g} / \mathrm{l}$. Calabrese and Mallek stated that the possibility of IACNS in a patient with a CSF count exceeding 200 cells $/ \mathrm{mm}^{3}$ is most unlikely. We could not find a value above 107 leucocytes $/ \mathrm{mm}^{3}$ in the literature. ${ }^{2-7}$ Our patient had a clinical picture suggestive of IACNS but the CSF cell count exceeded more than threefold the limit values mentioned above. The differential diagnosis could include vasculitis induced by bacterial infection or tuberculosis, although for the latter, the duration of the symptoms was too short and the glucose levels in the CSF were within normal limits. The cerebrovascular complications of bacterial menin-

1 Calabrese LH, Mallek JA. Primary angiitis of central nervous system. Report of 8 new cases, review of the literature, and proposal for diagnostic criteria. Medicine 1988; 67:20-39.

2 Oliveira V, Povoa P, Costa A, Ducla-Soares J. Cerebrospinal fluid and therapy of isolated angiitis of the central nervous fluid and therapy of isolated angitic

3 Abu-Shakra M, Khraishi M, Grosman H, Lewtas J, Cividino A, Keystone EC. Primary angiitis of the CNS diagnosed by angiography. $Q f$ Med $1994 ; 87: 351-8$.

4 Crane R, Kerr LD, Spiera H. Clinical analysis of isolated angiitis of the central nervous system. A report of 11 cases. Arch Intern Med 1991;151:2290-4.

\section{Learning points}

- isolated angiitis of the CNS usually manifests with progressive headache and ictal neurological deficits

- CSF examination may be normal or show inflammatory changes

- a pleocytosis of more than 500 leucocytes is uncommon but do not rule out this diagnosis

gitis are not so rare and may affect both large and small vessels of the CNS. The most frequent aetiology is Streptococcus pneumoniae, although any bacteria can be involved. ${ }^{8}$ In view of the possibility of bacterial meningitis with induced vasculitis, our patient received ceftriaxone and dexamethasone, but the negative culture suggested another diagnosis. The possibility of IACNS was considered and steroids were maintained at high doses, but an unfavourable outcome was not prevented.

We recommend that a diagnosis of IACNS should be considered in any patient with a suggestive clinical feature, even when the CSF examination points to a bacterial infection of the CNS.

5 Ozawa T, Sasaki O, Sorimachi T, Tanaka R Primary angii tis of the central nervous system: report of two cases and renew of the literature. Neurosurgery 1995;36:173-9.

6 Rhodes RH, Madelaire NC, Petrelli M, Cole M, Karaman BA. Primary angiitis and angiopathy of the central nervous system and their relationship to systemic giant cell arteritis. Arch Pathol Lab Med 1995;119:334-49.

7 Vanderzant C, Bromberg M, MacGuire A, McCune J. Isolated small-vessel angiitis of the central nervous system. Arch Neurol 1988;45:683-7.

8 Pfister H-W, Borasio GD, Dirnagl U, Bauer M, Einhaupl KM. Cerebrovascular complications of bacterial meningitis in adults. Neurology 1992;42:1497-504. 